\title{
Epidemiología del intento suicida en menores de 18 años atendidos en el Hospital Infantil los Ángeles, Pasto, Colombia
}

\author{
Epidemiology of suicidal intent in children under 18 attended in Los Angeles children's hospital in Pasto, Colombia
}

\author{
Freddy Israel Pantoja-Chamorro ${ }^{*}$ orcid.org/0000-0002-2856-9624 \\ Ana Ximena Rodríguez-Villota' orcid.org/0000-0001-7103-7386 \\ Jhuranny Urbano-Urbano' ${ }^{1}$ orcid.org/0000-0003-2191-6168 \\ Nancy Cabrera-Bravo' orcid.org/0000-0001-6985-1581
}

1 Hospital Infantil los Ángeles. Pasto, Colombia

Fecha de recepción: Marzo 6-2018 Fecha de revisión: Mayo 18 - 2018 Fecha de aceptación: Diciembre 11 - 2018

Pantoja-Chamorro F, Rodríguez-Villota AX, Urbano-Urbano J, Cabrera-Bravo N. Epidemiología del intento suicida en menores de 18 años atendidos en el Hospital Infantil los Ángeles, Pasto, Colombia. Univ. Salud. 2019;21(1):19-26. DOI: http://dx.doi.org/10.22267/rus.192101.136

\section{Resumen}

Introducción: El intento suicida es un problema de salud pública, las cifras aumentan a pesar de los programas preventivos, es necesario conocer su comportamiento en la población pediátrica y en la región. Objetivo: Caracterizar clínica y sociodemográficamente los menores de 18 años con intento suicida atendidos en el Hospital Infantil los Ángeles. Materiales y métodos: Estudio observacional, descriptivo de menores de 18 años con intento suicida (INSU) hospitalizados entre 2016 y 2017. Resultados: Se analizaron datos de 151 pacientes. Se encontró una prevalencia de 0,8 casos por mil ingresos. Falleció el 2\% (3) luego de ingesta de Paraquat ${ }^{\circledR}$. La edad promedio fue de 14,7 años, el $74 \%$ (112) fueron niñas. El 61,6\% (58) de la población con intento suicida tenía un ambiente familiar disfuncional. Los problemas académicos y la depresión se presentaron con alta frecuencia. Dentro de los principales mecanismos de INSU se evidenciaron: Cutting, consumo de antidepresivos tricíclicos, acetaminofén y organofosforados. Conclusiones: La prevalencia de INSU crece aceleradamente con respecto a registros reportados en años anteriores en la misma institución; el hecho puede reflejar problemas en los programas de salud dirigidos a la promoción de la salud mental del adolescente.

Palabras clave: Intento de suicidio; prevalencia; mortalidad; pediatría. (Fuente: DeCS, Bireme).

\begin{abstract}
Introduction: Suicide Attempt is a public health problem. Despite preventive programs, figures increase, so it is necessary to know its behavior in the pediatric population and in the region. Objective: To characterize clinically and socio-demographically children under 18 who presented suicidal intent and were attended at Los Angeles children's hospital. Materials and methods: An observational descriptive study was conducted with children under 18, who presented suicidal intent and were hospitalized between 2016 and 2017. Results: Data from 151 patients were analyzed. A prevalence of 0.8 cases per thousand incomes was found. $2 \%$ (3) died after Paraquat $₫$ intake. The average age was $14.7,74 \%$ (112) were girls. $61.6 \%$ (58) of the population with suicidal intent had a dysfunctional family environment. Academic problems and depression were presented with high frequency. Cutting, tricyclic antidepressants consumption, acetaminophen and organophosphorus were found among the main mechanisms of suicidal intent. Conclusions: The prevalence of suicidal intent grows rapidly with respect to records reported in previous years in the same institution. This fact may reflect problems in health programs aimed at promoting adolescent mental health.
\end{abstract}


Key words: Suicide, attempted; prevalence; mortality; pediatrics. (Source: DeCS, Bireme).

\section{Introducción}

El suicidio es un problema global que tiene impacto en todas y cada una de las esferas de la sociedad; según la Organización Mundial de la Salud (OMS) cada 40 segundos se produce una muerte por suicidio y cerca de 800.000 personas se suicidan cada año(1). Las cifras de suicidios e intentos de suicidio en la población colombiana adolescente $y$ ahora en niños también ha aumentado significativamente, constituyéndose en la segunda causa de muerte en el grupo de edad de 10 a 24 años; la tasa de intento suicida reportada en el Sistema Integral de Información SISPRO (Colombia) asciende de 0,9 por 100.000 habitantes en 2009 , a 2,05 por 100.000 habitantes en $2015^{(2,3)}$. Las estimaciones realizadas indican que en el mundo en 2020, las víctimas podrían ascender a 1,5 millones y de 10 a 20 veces más los intentos ${ }^{(4)}$.

La mayoría de los estudios indican que los casos de intento suicida en población infantil y adolescente van en aumento, convirtiéndose en un trágico problema de salud pública, con efectos devastadores emocionales, sociales y económicos sobre las familias, amigos y finalmente en la comunidad en general(2). De esta manera, es notable ver como en los hospitales del departamento de Nariño los casos de suicidio en la población infantil continúan presentándose a pesar de las medidas preventivas y de salud pública establecidas; el aumento de los casos es una característica que permite evaluar la efectividad de dichos programas, reflejando la necesidad de documentar este hallazgo ${ }^{(5)}$.

En los niños, la comprensión de la irreversibilidad de la muerte no está presente antes de los 8 años y los intentos de suicidio o intentos consumados son raros en menores de 12 años(6). En el grupo infantil, las decisiones son más impulsivas y se encuentran muy ligadas a características familiares, la interacción con el medio, los factores socio-ambientales, genéticos y propios de la niñez en consecuencia al desarrollo formativo de las funciones cerebrales superiores y la inmadurez de las conexiones cerebrales; a diferencia de los adolescentes mayores, en los que la presencia de trastornos mentales psiquiátricos, afectivos y sobretodo consumo de sustancias adictivas tienen mayor valor(6). La ideación suicida que es más frecuente en niños, no necesariamente se ve asociada con rasgos de trastornos psiquiátricos y afectivos ${ }^{(6,7) \text {. }}$

El presente estudio busca caracterizar la población menor de 18 años que ingresó al Hospital Infantil Los Ángeles con intento suicida, conocer qué tan vulnerable se encuentra el Departamento de Nariño por este flagelo, discriminando la población afectada de acuerdo a las áreas geográficas del Departamento, establecidas según el Plan participativo de Desarrollo Departamental(8) y determinar el comportamiento durante el periodo marzo 2016 diciembre 2017 con respecto al número de casos presentados. Este conocimiento se considera relevante por cuanto permite demostrar la situación actual de este evento en la región y en la institución, que se ha posicionado como uno de los hospitales acreditados en atención pediátrica en Colombia.

\section{Materiales y métodos}

\section{Tipo de estudio}

Se realizó un estudio observacional, descriptivo en menores de 18 años y mayores de un mes que ingresaron al Hospital Infantil los Ángeles de la ciudad de San Juan de Pasto, Nariño, con diagnóstico de intento suicida (INSU), definido en el manual diagnóstico y estadístico de trastornos mentales DSM 5, entre los años 2016 y $2017^{(9)}$. Se recolectaron los datos registrados en la ficha de vigilancia epidemiológica de intento suicida y las historias clínicas sistematizadas del Hospital Infantil teniendo en cuenta la descripción de variables clínicas y sociodemográficas tales como la edad, sexo, procedencia, régimen de seguridad social, etnia, escolaridad, funcionalidad de las relaciones intrafamiliares (según reporte de valoración psicológica en la historia clínica), problemas académicos, maltrato infantil, 
antecedente abuso sexual, antecedente familiar de INSU o suicidio, mecanismo del intento suicida, ingreso a cuidado intensivo y muerte. Se diseñó un instrumento de recolección de información tipo encuesta de 37 preguntas de opciones cerradas, para obtener los datos de las historias clínicas sistematizadas. Se validó por medio de la realización de una prueba piloto registrando y analizando los primeros 20 casos de dicho evento.

\section{Criterios de inclusión y exclusión}

Se controlaron sesgos de selección estableciendo criterios de inclusión: pacientes menores de 18 años de ambos sexos que ingresaron con diagnóstico de INSU y exclusión: pacientes que no tuvieran todos los datos dispuestos en el instrumento e información incompleta en la historia clínica. Los sesgos de información se controlaron realizando una búsqueda exhaustiva de historias clínicas por cada uno de los investigadores, se estandarizó las definiciones de las variables, se creó un proceso organizado para la revisión de las historias clínicas sin omitir información valiosa; se establecieron codificaciones en la toma de datos para mejorar su posterior procesamiento.

\section{Análisis de la información}

Los programas utilizados para la recolección, procesamiento y análisis de datos fueron $\operatorname{Excel}^{\circledR} \mathrm{y}$ el software estadístico SPSS ${ }^{\circledR}$ versión 21.

\section{Consideraciones éticas}

El proyecto lo avaló el comité de ética del Hospital Infantil los Ángeles de la ciudad de San Juan de Pasto, Colombia, el 29 de septiembre de 2017. Este estudio se clasificó como "sin riesgo" de acuerdo con el Artículo 11 de la Resolución 8430 de 1993. El manejo de la información sigue las normas de confidencialidad establecidas por el hospital, para garantizar el uso de los datos con fines científicos sin la divulgación de datos personales de los pacientes.

\section{Resultados}

\section{Variables sociodemográficas}

Se revisó un total 151 historias clínicas de menores de 18 años con intento suicida que consultaron e ingresaron al Hospital Infantil Los
Ángeles durante el periodo marzo 2016 diciembre 2017. Se excluyeron del estudio un total de 12 pacientes: 8 casos debido a que no eran diagnóstico INSU y 4 menores por registros incompletos. El diligenciamiento de obligatoriedad de la ficha de registro epidemiológico de intento de suicidio se consideró a partir de marzo de 2016, por lo tanto los datos de los meses previos no se tuvieron en cuenta.

Se encontró una prevalencia de intento suicida de 0,8 casos por mil ingresos. Los mayores picos de presentación se dieron en los meses de junio, agosto y noviembre, con menor frecuencia en enero y diciembre.

El porcentaje de niños de sexo masculino que manifestaron el intento suicida fue del 25,8\%; el 74,2 \% (112) pertenecían al sexo femenino. La edad promedio de intento suicida fue de 14,7 años, desviación estándar - ds $(1,9)$ años. En cuanto a la procedencia el 82,8\% (125) de los casos pertenecían a la zona urbana y la mayor parte de esta población con un $89,4 \%$ residía en la subregión del centro del departamento de Nariño con la mayor cantidad de individuos en la capital (Pasto) (10). La mayoría de los menores de edad con INSU estaban adscritos al Sistema General de Seguridad Social en Salud, predominando el régimen subsidiado con el 79,5\% (120), se encontró otro tipo de cobertura en salud como régimen contributivo con un menor porcentaje $(20,5 \%)$. Con respecto al nivel educativo el $76,2 \%$ de los pacientes cursaba secundaria, y el 13,2\% de esta población no cursaba ningún grado escolar (Tabla 1).

\section{Variables sociales}

El 61,6\% (58) de los pacientes con intento suicida tenía un ambiente familiar disfuncional; de acuerdo a la definición establecida previamente por el grupo de psicología del Hospital infantil Los Ángeles, basado en la aplicación del instrumento APGAR(10) (derivado de las siglas en inglés: Adaptation, Participation, Growth, Affection and Resources), el 9,9\% refirió haber sufrido maltrato infantil por parte de los padres o por algún miembro de la familia, el 5,3\% de los participantes tuvo antecedente de abuso sexual. 
Tabla 1. Características sociodemográficas

\begin{tabular}{llrr}
\hline Variable & & Frecuencia & \% \\
\hline Sexo & Masculino & 39 & 25,8 \\
& Femenino & 112 & 74,2 \\
\hline Etnia & Afrodescendiente & 4 & 2,6 \\
& Indígena & 6 & 4 \\
& Caucásico & 0 & 0 \\
& Mestizo & 141 & 93,4 \\
\hline Procedencia1 & Rural & 26 & 17,2 \\
& Urbano & 125 & 82,8 \\
\hline Procedencia2 & Centro & 135 & 89,4 \\
& Norte & 7 & 4,6 \\
& Pacífico & 5 & 3,3 \\
& Putumayo & 4 & 3,6 \\
\hline Seguridad social & Contributivo & 31 & 20,5 \\
& Subsidiado & 120 & 79,5 \\
\hline Escolaridad & Ninguno & 20 & 13,2 \\
& Primaria & 11 & 7,3 \\
& Secundaria & 115 & 76,2 \\
& Técnico & 5 & 3,3 \\
\hline Edad* & & 14,7 & $(1,9)$ \\
\hline Variables cuantitativas: Media, Desviación estándar & & \\
**Fuente: Plan Participativo de Desarrollo Departamental( 8$)$ &
\end{tabular}

Por otra parte, la valoración psicológica evidenció que existieron dificultades y problemas dentro de relaciones de noviazgo en los adolescentes en un $12,6 \%$. También se encontraron otros aspectos como: bullying en un $4 \%$, problemas académicos con un 41,1\%, consumo de sustancias psicoactivas en el $22,5 \%$, antecedente de intento suicida en algún familiar $6 \%$, y en mayor proporción se presentaron problemas de depresión en el 46\%. Del total de adolescentes estudiados el 36,4\% tuvieron el antecedente de intento suicida, (haber intentado por lo menos una vez quitarse la vida) (Tabla 2).

\section{Características clínicas}

El promedio de permanencia hospitalaria fue de 4,1 días con un promedio de costo hospitalario de 3.016 .000 pesos. El 7,9\% (12) de los pacientes ingresó a cuidado intensivo, de los cuales 5 presentaron lesión renal y 3 lesión hepática. El 42,4\% (64) de los pacientes recibió valoración por psiquiatría previa al alta al hogar. Fallecieron 3 pacientes que ingirieron Paraquat ${ }^{\circledR}$ (Tabla 3 ).
Tabla 2. Variables sociales

\begin{tabular}{|c|c|c|c|}
\hline Característica & & Frecuencia & $\%$ \\
\hline \multirow[t]{2}{*}{ Relación familiar } & Funcional & 58 & 38,4 \\
\hline & Disfuncional & 93 & 61,6 \\
\hline \multirow[t]{2}{*}{ Maltrato infantil } & No & 136 & 90,1 \\
\hline & $\mathrm{Si}$ & 15 & 9,9 \\
\hline \multirow{2}{*}{$\begin{array}{l}\text { Antecedente de abuso } \\
\text { sexual }\end{array}$} & No & 143 & 94,7 \\
\hline & $\mathrm{Si}$ & 8 & 5,3 \\
\hline \multirow[t]{2}{*}{ Problemas de pareja } & No & 132 & 87,4 \\
\hline & $\mathrm{Si}$ & 19 & 12,6 \\
\hline \multirow[t]{2}{*}{ Bullying } & No & 145 & 96 \\
\hline & $\mathrm{Si}$ & 6 & 4 \\
\hline \multirow{2}{*}{$\begin{array}{l}\text { Antecedente familiar } \\
\text { de intento suicida o } \\
\text { suicidio }\end{array}$} & No & 142 & 94 \\
\hline & $\mathrm{Si}$ & 9 & 6 \\
\hline \multirow{2}{*}{$\begin{array}{l}\text { Problemas } \\
\text { académicos }\end{array}$} & No & 89 & 58,9 \\
\hline & $\mathrm{Si}$ & 62 & 41,1 \\
\hline \multirow{2}{*}{$\begin{array}{l}\text { Intento de suicidio } \\
\text { previo }\end{array}$} & No & 96 & 63,6 \\
\hline & $\mathrm{Si}$ & 55 & 36,4 \\
\hline \multirow[t]{2}{*}{ Depresión } & No & 80 & 53 \\
\hline & $\mathrm{Si}$ & 71 & 47 \\
\hline \multirow[t]{2}{*}{ Trastorno bipolar } & No & 147 & 97,4 \\
\hline & $\mathrm{Si}$ & 4 & 2,6 \\
\hline \multirow[t]{2}{*}{ Esquizofrenia } & No & 149 & 98,7 \\
\hline & $\mathrm{Si}$ & 2 & 1,3 \\
\hline \multirow[t]{2}{*}{ Consumo de spa } & No & 117 & 77,5 \\
\hline & $\mathrm{Si}$ & 34 & 22,5 \\
\hline
\end{tabular}

Tabla 3. Características clínicas

\begin{tabular}{llrr}
\hline Característica & & Frecuencia & \% \\
\hline Ingreso a cuidado intensivo & $\mathrm{No}$ & 139 & 92,1 \\
& $\mathrm{Si}$ & 12 & 7,9 \\
\hline Lesión renal & $\mathrm{No}$ & 146 & 96,7 \\
& $\mathrm{Si}$ & 5 & 3,3 \\
\hline Lesión de hígado & $\mathrm{No}$ & 148 & 98 \\
& $\mathrm{Si}$ & 3 & 2 \\
\hline Valoración por psiquíatra & $\mathrm{No}$ & 87 & 57,6 \\
& $\mathrm{Si}$ & 64 & 42,4 \\
\hline Muerto & $\mathrm{No}$ & 148 & 98 \\
& $\mathrm{Si}$ & 3 & 2 \\
\hline Días de estancia* & \multicolumn{3}{c}{$4,1(4,8)$} \\
*Variables cuantitativas: Media, Desviación estándar
\end{tabular}

\section{Mecanismos del intento suicida (INSU)}

Dentro de los principales mecanismos de INSU se evidenció el cutting con un 35,1\% (53 casos). El 6,6\% (10 casos) presentó ahorcamiento, 6,6\% (10 
casos) consumo de organofosforados y otros mecanismos (22\%) (34 casos) (lanzarse a un carro o desde edificios, consumo de hipoclorito de sodio, cera, pegante bóxer o alcohol) y medicamentos (35\%) (53 casos) (anticonceptivos, metocarbamol, omeprazol, antibióticos, antihipertensivos, medicamentos naturistas). El 6,6\% (10 casos) consumió antidepresivos tricíclicos y 10 casos acetaminofén. El 2,6\% (4 casos) consumió Paraquat $^{\circledR}$, falleciendo 3 de ellos (2\%). (Tabla 4).

Tabla 4. Mecanismos y sustancias ingeridas usadas para el INSU

\begin{tabular}{|c|c|c|c|}
\hline Característica & & Frecuencia & $\%$ \\
\hline \multirow[t]{2}{*}{ Antidepresivos } & No & 138 & 91,4 \\
\hline & $\mathrm{Si}$ & 13 & 8,6 \\
\hline \multirow[t]{2}{*}{ Antipsicótico } & No & 149 & 98,7 \\
\hline & $\mathrm{Si}$ & 2 & 1,3 \\
\hline \multirow[t]{2}{*}{ Acetaminofén } & No & 141 & 93,4 \\
\hline & $\mathrm{Si}$ & 10 & 6,6 \\
\hline \multirow[t]{2}{*}{ Otros medicamentos } & No & 98 & 64,9 \\
\hline & $\mathrm{Si}$ & 53 & 35,1 \\
\hline \multirow[t]{2}{*}{ Organofosforados } & No & 141 & 93,4 \\
\hline & $\mathrm{Si}$ & 10 & 6,6 \\
\hline \multirow[t]{2}{*}{ Carbamatos } & No & 149 & 98,7 \\
\hline & $\mathrm{Si}$ & 2 & 1,3 \\
\hline \multirow[t]{2}{*}{ Paraquat $^{\circledR}$} & No & 147 & 97,4 \\
\hline & $\mathrm{Si}$ & 4 & 2,6 \\
\hline \multirow[t]{2}{*}{ Warfarínico } & No & 150 & 99,3 \\
\hline & $\mathrm{Si}$ & 1 & 0,7 \\
\hline \multirow[t]{2}{*}{ Ahorcamiento } & No & 141 & 93,4 \\
\hline & $\mathrm{Si}$ & 10 & 6,6 \\
\hline \multirow[t]{2}{*}{ Cutting } & No & 98 & 64,9 \\
\hline & $\mathrm{Si}$ & 53 & 35,1 \\
\hline \multirow[t]{2}{*}{ Otros mecanismos } & No & 117 & 77,5 \\
\hline & $\mathrm{Si}$ & 34 & 22,5 \\
\hline
\end{tabular}

\section{Discusión}

Según el Instituto Nacional de Salud para el año de 2017, la situación de Colombia refleja un aumento en la tasa del intento suicida y suicidio de manera progresiva, y Nariño ocupa el quinto lugar como uno de los departamentos con mayores tasas de intento suicida en Colombia(4).
Este estudio pretende evidenciar un problema de salud pública en auge que afecta a la población, por el notable aumento de casos de intento de suicidio que ingresaron al Hospital Infantil, comparado con años previos y reportados en estudios como los de Eraso et al.(11), en 2007 con 50 casos en 1 año, Jiménez et al.(5), con 100 casos en 6 años y Guerrero(12) con 97 pacientes en 5 años; esta situación es preocupante ya que en esta ocasión se reportaron 161 casos en 21 meses. Además de las cifras en ascenso, se constata que los datos de variables como el sexo femenino, el promedio de edad, la disfunción familiar y la depresión son similares; por el contrario el cutting y ahorcamiento no se evidencian en dichas publicaciones, sin embargo, este último es reportado en estudios mexicanos como el principal mecanismo de suicidio en menores de 20 años(13,14).

Con respecto al intento suicida, se encontró que el género femenino fue el más afectado con un $74,2 \%$, resultado que coincide con reportes de la Academia Americana de Pediatría y estudios como el de Sánchez-Loyo(15) de la ciudad de México, en donde se observó que la prevalencia de intento suicida en población adolescente fue mucho más alta en mujeres $(6,37 \%)$ con respecto a la de los hombres $(2,14 \%)$, situación que sigue sustentando que los varones presentan métodos más letales al momento de intentar suicidarse. El promedio de edad de esta población se encontró en 14,7 años y el 82,8\% provino del área urbana, resultados similares que fueron encontrados en un estudio realizado por la Universidad Nacional en la ciudad de Manizales Colombia(7). La población de estudio afiliada al régimen subsidiado presentó mayor frecuencia de intento suicida con un $79,5 \%$, dato novedoso, teniendo en cuenta que es una variable no estudiada en otras investigaciones.

Factores como la disfunción familiar, las dificultades académicas, el antecedente personal de INSU, la depresión y los problemas con la pareja fueron los de mayor frecuencia; esta situación demuestra que el ambiente social juega un papel importante en la generación y el impulso del evento suicida, por lo que sería importante desarrollar investigaciones que permitan 
esclarecer con mejor evidencia científica cómo afectan las variables psicosociales la presencia de este problema de salud pública; además, cómo influyen en el estado psicológico del adolescente ya que el estado depresible podría llegar a ser una variable intermedia en este mapa tan complejo donde se pueden considerar múltiples factores disparadores(16-20).

Se propone un modelo teórico en donde la estructura familiar opere como eje fundamental en el desarrollo del pensamiento y el actuar del adolescente, obviamente influenciado por factores externos, que facilite el desarrollo de las conductas y pensamientos suicidas. El ser humano desde su niñez y durante los primeros 5 años, tiene la vulnerabilidad de ser "moldeado" con los aprendizajes y aportes por parte de su entorno familiar, generando ciertas habilidades y capacidades psicosociales que permiten afrontar de la mejor manera las adversidades que le propone la vida(21).

La adolescencia siendo una etapa difícil y de afrontamiento y reconocimiento de sí mismo requiere del apoyo y acompañamiento de figuras paternales serias, responsables y comprometidas de modo que factores como el alcoholismo y la drogadicción no afecten el curso normal de esta etapa; se sabe entonces que si este eje fundamental llamado "familia" falla por diversas situaciones críticas, el adolescente sería más vulnerable a tomar conductas inapropiadas como el intento suicida(18,19,22). Además, debe ser prioritario trabajar de manera preventiva en escenarios como el hogar y la escuela en tres esferas clave del comportamiento humano para contrarrestar la depresión en los niños y adolescentes incluyendo "la persona, lo social y lo familiar" $(3,23)$.

Por otra parte, la fácil adquisición de sustancias tóxicas en el mercado y en el hogar generan un riesgo potencial para la población pediátrica, por esto, se hace necesario controlar de una manera estricta este hecho por partes de las autoridades de salud pública. Asimismo, mencionar que los mecanismos de intento suicida encontrados incluyen una variedad de métodos que no se evidencian en otros estudios, con el agravante de que pueden llegar a ser muy letales (ingestión de venenos, diferentes tipos de medicamentos, antibióticos, lanzamiento hacia vehículos y desde las alturas) en comparación a otros estudios que soportan la ingestión de tóxicos como el principal mecanismo, como el publicado por Castaño et al(7).

Se destaca que el cutting fue el segundo mecanismo más evidenciado en la población de estudio, variable descrita en otras revisiones bibliográficas con menor frecuencia. Asimismo, se observó aumento en la frecuencia de los casos de ahorcamiento, que en otros estudios se da en menor número(11). Aunque el artículo se basa en el intento suicida, la letalidad de los métodos en las mujeres es una variable a considerar ya que 3 de los 4 pacientes que ingirieron Paraquat $^{\circledR}$ fallecieron, siendo las 3 de sexo femenino, situación preocupante que demuestra la alta letalidad de esta sustancia sumada a la fácil disponibilidad de la misma en el mercado.

De acuerdo a la valoración inicial realizada por psicología, el 42,4\% de los pacientes requerían atención por psiquiatría, los demás fueron remitidos a este servicio de manera ambulatoria. Es fundamental que al paciente con un primer intento suicida se le garantice seguimiento e intervención oportunos por parte de un equipo multidisciplinario competente, por cuanto existe 18 veces más probabilidades de intento en el año siguiente, así como se ha demostrado que el intento suicida y las autolesiones graves son factores de riesgo importantes que determinan el comportamiento suicida(24-27). El 7,9\% de los pacientes requirieron ingreso a la Unidad de Cuidado Intensivo Pediátrica, dato a tener en cuenta ya que la gravedad de los eventos genera mayor estancia hospitalaria, mayores costos al sistema, alta comorbilidad, pérdida de días escolares en los niños y laborales en los padres y acudientes ${ }^{(2,4)}$.

\section{Conclusiones}

Se determinó que el costo de la hospitalización de los adolescentes por INSU es en promedio $\$ 3.016 .000$. Tal característica no se encontró como variable analizada en los estudios de referencia para este trabajo, pero siempre será 
importante cuantificar económicamente las situaciones o eventos de salud pública, pensando en que este tipo de inversiones se podrían transferir a los programas de prevención.

Si bien el diseño del estudio presenta resultados descriptivos, vale la pena destacar las altas frecuencias de características que pueden mostrar el vínculo del ambiente social y familiar con el intento suicida, razón por la cual los diseños de estudios futuros podrían enfocarse en esclarecer estos factores y así evaluar la necesidad de trabajar de manera oportuna y eficaz en programas de salud pública que incluyan el fortalecimiento de la estructura familiar y la identificación temprana del riesgo suicida en escenarios como el hogar, la escuela y centros de salud.

Es preocupante conocer como la frecuencia de estos eventos viene en aumento en la población infantil reflejando y descubriendo las falencias en los programas de salud pública ya que no solo se están perdiendo vidas si no años de vida saludable, gastando recursos humanos, físicos y económicos cuando ya los problemas se han desarrollado y dejando de invertir estos mismos en la prevención y educación de la población. Igualmente se hace énfasis en la preocupación que existe por los países de Latinoamérica con respecto al uso de pesticidas tóxicos, altamente letales y de fácil disponibilidad por lo que es necesario evaluar las políticas ambientales y de salud en cuanto a la restricción de sustancias como el Paraquat ${ }^{\circledR}$, tan peligrosa y mortal con cantidades ingeridas tan pequeñas como de una onza.

Es necesario que luego de la presentación del intento suicida, las valoraciones, intervenciones y seguimiento de parte del personal de psicología y psiquiatría sean efectivas y oportunas ya que una vez presentado el evento está demostrado que existen altas probabilidades de volverse a repetir.

Este tipo de estudio únicamente permite explorar algunas características de la población pediátrica que ingresó al hospital. Considerando su diseño se debe tener en cuenta que existen algunas limitaciones como el hecho de que solo se evaluaron los pacientes que llegaron al Hospital Infantil Los Ángeles, no se tuvieron en cuenta otros centros hospitalarios, sin embargo éste es la institución de referencia en Pediatría para los departamentos de Nariño y Putumayo; no se relacionaron variables que pudieran demostrar asociación o relación con variables resultado.

\section{Agradecimientos}

Al Hospital Infantil Los Ángeles de la ciudad de Pasto, Colombia.

\section{Conflicto de intereses}

Ninguno a declarar por los autores.

\section{Referencias}

1. Organización Mundial de la Salud. Suicidio. Ginebra: OMS; $2018 . \quad$ Disponible en: http://www.who.int/es/news-room/factsheets/detail/suicide

2. De la Hoz F, Martínez Duran M, Pacheco García OE, Alejandro Alfonso C, Blandón Rodríguez AM. Protocolo de Vigilancia en Salud Pública: Intento de Suicidio. Bogotá: Inst Nac Salud; 2014

3. Mosquera L. Conducta suicida en la infancia: Una revisión crítica. Rev Psicol Clínica con Niños y Adolesc [Internet]. 2016;3(1):9-18. Available from: https://dialnet.unirioja.es/servlet/articulo? codigo $=528$ 8635\&info=resumen\&idioma $=$ SPA

4. Ministerio de Salud y la Protección Social. Boletín de salud mental, conducta suicida. Bogotá: MinSalud; 2017. Available

from: https://www.minsalud.gov.co/sites/rid/Lists/Bibliotec aDigital/RIDE/VS/PP/ENT/boletin-conductasuicida.pdf

5. Jiménez-Quenguan M, Hidalgo-Bravo J, Camargo Santacruz C, Dulce Rosero PB. El intento de suicidio en la población pediátrica, una alarmante realidad. Rev Ciencias la Salud. 2014;12(1):59-92.

6. Macana N. Comportamiento del suicidio en Colombia, 2011. Forensis [Internet]. 2012;13(1):243-66. Available from:

http://fpv.org.co/images/repositorioftp/capitulo1y7.p df

7. Alzate L, Bentacur MB, Castaño J, Cañon S. Factor de riesgo suicida según dos cuestionarios y factores asociados en estudiantes de la Universidad Nacional de Colombia sede Manizales. Divers.: Perspect. Psico. 2012;11(2):193-205.

8. Gobernación de Nariño. Plan Participativo del Desarrollo Departamental. Nariño: Gobernación de Nariño; 2016. Disponible en: http://xn--nariorta.gov.co/inicio/files/PlanDesarrollo/Plan_De_Desarro llo_Nario_corazn_Del_Mundo.pdf

9. Asociación Americana de Psiquiatría (APA). Actualización del DSM-5® septiembre 2016. Man 
diagnósrico y estadístico Trastor Ment [Internet]. 2016;15-21. Available from: http://psychiatryonline.org/pbassets/dsm/update/Spanish_DSM5Update2016.pdf

10. Suarez Cuba MÄAEM. Apgar Familiar : Una Herramienta Para Detectar Disfunción Familiar. Rev Médica La Paz. 2014;20(1):53-7.

11. Osejo J, Camayo D, Patiño P. Caracterización de los pacientes con intento de suicidio atendidos en un hospital pediátrico. Univ. Salud [Internet]. 2008;1(10):18-31. Available from: http://revistas.udenar.edu.co/index.php/usalud/articl e/view/220

12. Guerrero-Martínez LF. Descripción del intento de suicidio como constructo de violencia en la población pediátrica femenina. Rev. Fac. Nac. Salud Pública [Internet]. 2016;34(3):306-315 Available from: http://www.scielo.org.co/pdf/rfnsp/v34n3/0120386X-rfnsp-34-03-00306.pdf

13. Sánchez-Cervantes FS, Serrano-González RE, MárquezCaraveo ME. Suicidios en menores de 20 años. México 1998-2011. Salud Ment. 2015;38(5):379-89.

14. Shain B. Suicide and Suicide Attempts in Adolescents. Pediatrics. 2016;138(1): e20161420.

15. Sánchez-Loyo LM, Morfín-López T, García de Alba García JE, Quintanilla-Montoya R, Hernández-Millán R, et al. Suicide Attempts in Mexican Teenagers: a Cultural Consensus Theory Perspective. Acta Investig psicológica [Internet]. 2014;4(1):1446-58. Available from: http://www.scielo.org.mx/scielo.php?script=sci_arttext \&pid=S200748322014000100010\&lng=en\&nrm=iso\&tlng=en

16. Siabato-Macías EF, Forero-Mendoza IX, SalamancaCamargo Y. Asociación entre depresión e ideación suicida en un grupo de adolescentes colombianos. Pensamiento Psicológico [Internet]. 2017;15(1):51-61. doi:10.11144/Javerianacali.PPSI15-1.ADIS.

17. Pérez-Olmos I, Rodríguez-Sandoval E, Dussán-Buitrago MM, Ayala-Aguilera JP. Caracterización Psiquiátrica y Social del Intento Suicida Atendido en una Clínica Infantil, 2003-2005. Rev salud pública [Internet]. 2007;9(2):230-40. Disponible en: http://www.scielo.org.co/pdf/rsap/v9n2/v9n2a07.pdf

18. Bittencourt ALP, França LG, Goldim JR. Adolescência vulnerável: fatores biopsicossociais relacionados ao uso de drogas. Rev Bioética [Internet]. 2015;23(2):311-9. Available from: http://www.scielo.br/scielo.php?script=sci_arttext\&pi $\mathrm{d}=$ S1983-80422015000200311\&lng=pt\&tlng=pt

19. Castell-Florit Serrate P. Editorial. Rev Cuba Med Gen Integr. 2017;24(1):281-95.

20. Rodway C, Tham SG, Ibrahim S, Turnbull P, Windfuhr K, Shaw J, et al. Suicide in children and young people in England: a consecutive case series. Lancet Psychiatry [Internet]. 2016;3(7):751-9. Available from: http://dx.doi.org/10.1016/S2215-0366(16)30094-3

21. Caballo VE, Simón MÁ. Manual de psicología clínica infantil y del adolescente: trastornos generales transtornos específicos. Madrid: Editorial Piramide; 2002.
22. Medina-Pérez ÓA, Díaz-Téllez ÁS, Rozo-David AJ. Characterization of suicide in antioquia, Colombia adolescents. 2000-2010. Rev Fac Med [Internet]. 2015;63(3):431-8. Available from: https://www.scopus.com/inward/record.uri?eid=2s2.084940658542\&partnerID $=40 \& \mathrm{md} 5=7 \mathrm{~b} 7 \mathrm{e} 7 \mathrm{fd} 6 \mathrm{~d} 94 \mathrm{ae} 3 \mathrm{~b}$ $6639663255 \mathrm{e} 65 \mathrm{ebfa}$

23. Chávez-Hernández AM, Correa-Romero FE, KleinCaballero AL, Macías-García LF, Cardoso-Espindola KV, Acosta-Rojas IB. Sintomatología Depresiva, Ideación suicida y Auto-concepto en una muestra de infantes mexicanos. Av en Psicol Latinoam [Internet]. 2017;35(3):501. Available from: https://revistas.urosario.edu.co/index.php/apl/article /view/4733

24. McKean AJS, Pabbati CP, Geske JR, Bostwick JM. Rethinking Lethality in Youth Suicide Attempts: First Suicide Attempt Outcomes in Youth Ages 10 to 24. J Am Acad Child Adolesc Psychiatry [Internet]. 2018;57(10):786-91. Available from: https://doi.org/10.1016/j.jaac.2018.04.021

25. Pugliese SV. Indicadores De Riesgo De Conducta Suicida En Una Muestra De Adolescentes Suicidal Behavior Risk Indicators in a Sample of Adolescents. Subj y ProceSoS Cogn. 2015;19:228-45.

26. Russi AE, Brown MA. Suicide Attempt as a Risk Factor for Completed Suicide: Even More Lethal Than We Knew. Am J Psychiatry. 2016;165(2):255-69.

27. Olfson M, Wall M, Wang S, Crystal S, Bridge JA, Liu S-M, et al. Suicide After Deliberate Self-Harm in Adolescents and Young Adults. Pediatrics [Internet]. 2018;141(4):e20173517. Available from: http://pediatrics.aappublications.org/lookup/doi/10.1 542/peds.2017-3517 